\title{
Splitting of Potential Curves in the Two-Coulomb-Centre Problem
}

\author{
Michal Hnatič ${ }^{1,2,3, \star}$, Viktor M. Khmara ${ }^{3, \star \star}$, Volodymyr Yu. Lazur ${ }^{4, \star \star \star}$, and \\ Oleksandr K. Reity ${ }^{4, \star \star \star \star}$ \\ ${ }^{1}$ Department of Theoretical Physics, SAS, Institute of Experimental Physics, Watsonova 47, 04001 Košice, \\ Slovakia \\ ${ }^{2}$ Peoples' Friendship University of Russia (RUDN University), 6 Miklukho-Maklaya St, Moscow, 117198, \\ Russian Federation \\ ${ }^{3}$ Faculty of Science, P. J. Šafárik University, Šrobárová 2, 04154 Košice, Slovakia \\ ${ }^{4}$ Department of Theoretical Physics, Uzhhorod National University, Voloshyna 54, 88000 Uzhhorod, Ukraine
}

\begin{abstract}
The quasiclassical expression for the exchange interaction $\Delta E$ of potential curves at the points of their quasicrossing in the two-Coulomb-centre problem is found. It can be used for the calculation of cross sections of charge exchange processes between hydrogen or hydrogen-like atoms and bare nuclei.
\end{abstract}

\section{Introduction}

In order to solve many problems arising in the theory of slow atomic collisions, e.g. for the calculation of the matrix element $\Delta(R)$ of the exchange interaction between a hydrogen atom (or H-like ion) and a bare nucleus, it is necessary to know the two-centre radial and angular Coulomb spheroidal wave functions (CSWF) [1]. In the general non-resonant case $\left(Z_{1} \neq Z_{2}\right)$, the exchange matrix element $\Delta(R)$ was determined formerly $[2,3]$ under the condition requiring that the two-Coulomb-centre spheroidal wave function reach the one-centre parabolic wave function when an electron approaches one of the nuclei. The correct result for the exchange energy splitting $\Delta E=2 \Delta(R)$ at the quasicrossing points can actually be obtained only (see [4]) when the wave functions of the zeroth order approximation are considered in the spheroidal system of coordinates. The fact is that the exchange matrix element $\Delta(R)$ is defined by the asymptotic region of electron coordinates where the one-centre parabolic and spheroidal wave functions of a hydrogen atom differ substantially from each other. To be more specific, at large distances from the nucleus, a set of several Coulomb parabolic wave functions makes a contribution to the asymptotic behaviour of CSWF. This circumstance makes it difficult to apply the comparison equation method $[1,5]$ to determine the mentioned asymptotic behaviour (see, for instance, [4]).

In this paper, we provide a combined approach to the solution of the quantum two-Coulombcentre $Z_{1} e Z_{2}$ problem. The slightly modified perturbation theory has been used to determine the

\footnotetext{
$\star$ e-mail: hnatic@saske.sk

$\star \star$ e-mail: viktor.khmara@student.upjs.sk

$\star \star \star$ e-mail: volodymyr.lazur@uzhnu.edu.ua

$\star \star \star \star$ e-mail: okreity@gmail.com
} 
local behaviour of the solutions of the $Z_{1} e Z_{2}$ problem near a certain nucleus [6, 7]. We propose to employ the quasiclassical approach (the WKB method) to construct the asymptotic expansions of the angular CSWF in the internuclear region. In contrast to the perturbation theory, the quasiclassical approximation is not restricted by the smallness of the interaction and, hence, has a wider applicability domain, allowing the qualitative description for the behaviour and properties of quantum mechanical systems. Using the obtained quasiclassical CSWF and the Firsov surface integral method [8], we calculate the first three terms of the asymptotic behaviour of the exchange interaction potential of an ion with an atom for the general nonresonance case.

\section{The wave functions of the two-Coulomb-centre problem}

The Schrödinger equation of the $Z_{1} e Z_{2}$ problem after separation of the variables in the prolate spheroidal coordinates $(\xi, \eta, \phi)$, using the new variables $\mu=(\xi-1) R / 2, v=(1+\eta) R / 2$ and replacing the wave function by the product

$$
\Psi(\vec{r}, R)=\frac{U(\xi, R)}{\sqrt{\xi^{2}-1}} \frac{V(\eta, R)}{\sqrt{1-\eta^{2}}} \frac{e^{ \pm i m \phi}}{\sqrt{2 \pi}}=\frac{\psi(\xi, \eta, R)}{\sqrt{\left(\xi^{2}-1\right)\left(1-\eta^{2}\right)}} \frac{e^{ \pm i m \phi}}{\sqrt{2 \pi}},
$$

results in the following system of differential equations $(\gamma=\sqrt{-2 E})$ :

$$
\begin{aligned}
& U^{\prime \prime}(\mu)-\left[\gamma^{2}-\frac{Z_{1}+Z_{2}+\lambda_{\xi} / R}{\mu}-\frac{Z_{1}+Z_{2}-\lambda_{\xi} / R}{R+\mu}+\frac{R^{2}\left(m^{2}-1\right)}{4 \mu^{2}(R+\mu)^{2}}\right] U(\mu)=0, \\
& V^{\prime \prime}(v)-\left[\gamma^{2}-\frac{Z_{1}-Z_{2}-\lambda_{\eta} / R}{v}+\frac{Z_{1}-Z_{2}+\lambda_{\eta} / R}{R-v}+\frac{R^{2}\left(m^{2}-1\right)}{4 v^{2}(R-v)^{2}}\right] V(v)=0
\end{aligned}
$$

with boundary conditions $U(0)=U(\infty)=0$ and $V(0)=V(R)=0$.

The solutions of the equations (2), (3) for $\mu \ll R$ (in the area near the internuclear axis) and $v \ll R$ (close to the $Z_{1}$ centre) can be obtained by means of the perturbation theory. The details of such a calculation can be found in $[6,7]$, here we give only the final result:

$$
\begin{gathered}
\psi^{\text {pert }}(\mu, v, R)=C(R) U^{\text {pert }}(\mu) V^{\text {pert }}(v), \\
U^{\text {pert }}=f_{n_{1}}^{(0)}(\mu)+\sum_{p=1}^{3} \sum_{k=-p}^{p} c_{n_{1}+k}^{(p)} f_{n_{1}+k}^{(0)}(\mu), \quad V^{\text {pert }}=f_{n_{2}}^{(0)}(v)+\sum_{p=1}^{3} \sum_{k=-p}^{p} c_{n_{2}+k}^{(p)} f_{n_{2}+k}^{(0)}(v), \\
f_{n_{i}}^{(0)}(x)=\left[\frac{\left(n_{i}+m\right) !}{n_{i} !(m !)^{2}\left(2 n_{i}+m+1\right)}\right]^{1 / 2}(2 \gamma x)^{(m+1) / 2} \mathrm{e}^{-\gamma x} F\left(-n_{i}, m+1,2 \gamma x\right),
\end{gathered}
$$

where $F\left(-n_{i}, m+1,2 \gamma x\right)$ is the confluent hypergeometric function and the coefficients $C(R)$ and $c_{n_{i}+k}^{(p)}$ $(p=1,2,3)$ were found in $[6,7]$.

In the under-barrier region, the wave function of an electron moving in the field of well-separated nuclei (i.e., when $R$ is large) is mostly concentrated near the internuclear axis, exponentially vanishing with the increase of $\mu$. Considering this fact, the quasiradial wave function $U^{\text {pert }}(\mu)$ seems to be fairly accurate here. An entirely different situation occurs for the quasiangular function: when an electron moves from the first nucleus to the second one (and $v$ changes from 0 to $R$ ), the influence of $Z_{1}$ nucleus is gradually replaced by the influence of $Z_{2}$. The perturbation theory is not sufficient in this area of the under-barrier electron motion. Therefore, we propose to use the quasiangular WKB-function [6, 7]

$$
V^{\text {quas }}=\frac{C_{0}}{\sqrt{q}} \exp \left[-\int_{v_{2}}^{v} q\left(v^{\prime}\right) d v^{\prime}+S_{1}+S_{2}+\mathrm{O}\left(R^{-4}\right)\right], q(v)=\sqrt{\gamma^{2}-\frac{2 \tilde{Z}_{1}}{v}-\frac{2 \tilde{Z}_{2}}{R-v}+\frac{\left(m^{2}-1\right)}{4 v^{2}(1-v / R)^{2}}},
$$


where the quasiclassical corrections $S_{1}$ and $S_{2}$ are determined by the formulae

$$
\begin{aligned}
S_{1}= & -\frac{\tilde{Z}_{1}}{4 \gamma^{3} v^{2}}\left(1+\frac{17 \tilde{Z}_{1}}{6 \gamma^{2} v}\right)+\frac{\tilde{Z}_{1} \tilde{Z}_{2}}{2 \gamma^{5} R^{3}} \ln \frac{v}{R-v}+\frac{m^{2}-1}{16 \gamma^{3}}\left(\frac{1}{v^{3}}+\frac{1}{v^{2} R}-\frac{1}{R(R-v)^{2}}-\frac{1}{(R-v)^{3}}\right) \\
& +\frac{\tilde{Z}_{1} \tilde{Z}_{2}}{4 \gamma^{5} R}\left(\frac{3}{(R-v)^{2}}-\frac{3}{v^{2}}+\frac{1}{R}\left[\frac{1}{R-v}-\frac{1}{v}\right]\right)+\frac{\tilde{Z}_{2}}{4 \gamma^{3}(R-v)^{2}}\left(1+\frac{17 \tilde{Z}_{2}}{6 \gamma^{2}(R-v)}\right)+C_{1}, \\
S_{2}= & \frac{\tilde{Z}_{1}}{4 \gamma^{4} v^{3}}+\frac{\tilde{Z}_{2}}{4 \gamma^{4}(R-v)^{3}}+C_{2}, \quad \tilde{Z}_{1,2}=\left[ \pm\left(Z_{1}-Z_{2}\right)-\lambda_{2} / R\right] / 2 .
\end{aligned}
$$

The constants $C_{0}, C_{1}$ and $C_{2}$ were found by the exact matching of $V^{\text {quas }}$ (7) with $V^{\text {pert }}$ from (5). Their explicit expressions are given in [7]. Note also that the expression for $C_{0}$ contains a misprint: the additional factor $\sqrt{\gamma}\left[n_{2} !\left(n_{2}+m\right) !\left(2 n_{2}+m+1\right)\right]^{-1 / 2}$ was omitted there.

Considering the quasiangular function (7), the final expression for the wave function centred on the atom $\left(e, Z_{1}\right)$ is of the form

$$
\psi(\mu, \nu, \phi)=C(R) \frac{R^{2}}{4} \frac{U^{\text {pert }}(\mu)}{\sqrt{\mu(R+\mu)}} \frac{V^{\text {quas }}(v)}{\sqrt{v(R-v)}} \frac{e^{ \pm i m \phi}}{\sqrt{2 \pi}} .
$$

\section{Splitting of the potential curves at the quasicrossing points}

The calculation of the exchange splitting $\Delta E(R)$ of the potential curves at the quasicrossing points can be carried out using the well-known surface integral formula [8]

$$
\Delta E=\oint_{S}\left(\Psi_{I}^{*} \vec{\nabla} \Psi_{I I}-\Psi_{I I}^{*} \vec{\nabla} \Psi_{I}\right) \cdot d \vec{S}
$$

Here $\Psi_{I}$ and $\Psi_{I I}$ are the electron wave functions of the quasimolecule $\left(Z_{1}, e, Z_{2}\right)$. In the separated atoms limit $(R \rightarrow \infty)$ those turn into the wave function of the hydrogen-like atom $\left(e, Z_{1}\right)$ and of the ion (e, $\left.Z_{2}\right)$, respectively; $d \vec{S}=\vec{n} d S ; S$ is the surface enclosing the half-space containing nuclei $Z_{1} ; \vec{n}$ is the surface vector normal to $S$. As an integration surface in (11) we have chosen the paraboloid of revolution $\eta=$ const. Omitting the details of this calculation, we provide only the resulting formula for $\Delta E$ :

$$
\begin{array}{r}
\Delta E=\frac{2 \gamma^{2}(-1)^{n_{2}+n_{2}^{\prime}}(2 \gamma R)^{n_{2}+n_{2}^{\prime}+m+1} \mathrm{e}^{-\gamma R}}{\left[n n^{\prime} n_{2} !\left(n_{2}+m\right) ! n_{2}^{\prime} !\left(n_{2}^{\prime}+m\right) !\right]^{1 / 2}}\left\{1-\frac{1}{2 \gamma R}\left[\frac{A_{2}^{2}+A_{2}^{\prime 2}}{4}+A_{2} A_{2}^{\prime}+\frac{1-m^{2}}{2}\right]\right. \\
-\frac{A_{2}+A_{2}^{\prime}}{2 \gamma R}-\frac{A_{1}}{2 \gamma^{2} R}\left(\frac{Z_{1}}{n}+\frac{Z_{2}}{n^{\prime}}\right)+\frac{\left[A_{2}^{2}+A_{2}^{\prime 2}+4 A_{2} A_{2}^{\prime}+2\left(1-m^{2}\right)\right]^{2}}{128 \gamma^{2} R^{2}}+\frac{A_{1}\left(3 A_{1}+1-m^{2}\right)}{4 \gamma^{2} R^{2}} \\
\left.+\frac{A_{2}^{3}+A_{2}^{\prime 3}+\left(A_{2} A_{2}^{\prime}-4 A_{1}+2 m^{2}-6\right)\left(A_{2}+A_{2}^{\prime}\right)}{32 \gamma^{2} R^{2}}+\frac{\left(A_{1}-1\right)\left(A_{2}+A_{2}^{\prime}\right)^{2}+2 A_{2} A_{2}^{\prime}\left(A_{1}-2\right)}{8 \gamma^{2} R^{2}}\right\} .
\end{array}
$$

Here $A_{i}=2 n_{i}+m+1, A_{i}^{\prime}=2 n_{i}^{\prime}+m+1$. In the resonance case $Z e Z$, formula (12) gives the difference between the energies of gerade and ungerade states in agreement with the results of $[3,9]$. It should be noted also that the formulae for $H_{2}^{+}$in $[1,3]$ contain a mistake in the terms of the order of $R^{-2}$.

Concerning the splitting of the potential curves obtained by means of the comparison equation method, it should be noted that various formulae for the splitting of potential curves at the quasicrossing points are available. These can be written compactly as follows:

$$
\begin{gathered}
\Delta E=T_{\{\mathrm{KS}, \mathrm{P}, \mathrm{PKS}\}} \delta\left(n_{2}, n_{2}^{\prime}, m, p\right), \\
T_{\mathrm{KS}}=2 \frac{\left(Z_{2}-Z_{1}\right)^{2}}{\left(n_{2}^{\prime}-n_{2}\right)^{3}}, \quad T_{\mathrm{P}}=\frac{\partial E_{1}}{\partial n_{2}}+\frac{\partial E_{2}}{\partial n_{2}^{\prime}}, \quad T_{\mathrm{PKS}}=\frac{\partial E_{1} / \partial n_{2}}{\sqrt{1+\frac{\partial \beta}{\partial E_{1}} \frac{\partial E_{1}}{\partial n_{2}}}}+\frac{\partial E_{2} / \partial n_{2}^{\prime}}{\sqrt{1-\frac{\partial \beta}{\partial E_{2}} \frac{\partial E_{2}}{\partial n_{2}^{\prime}}}},
\end{gathered}
$$


where $\delta$ is determined by formula (4.36) from [1]. Komarov and Slavyanov [2] first proposed to determine $T$ by differentiating the expression $E=-\left(Z_{2}-Z_{1}\right)^{2} /\left(2 \beta^{2}\right)$ with respect to the indices, giving $T=T_{\mathrm{KS}}$. Power [3] criticized this expression and noted that it does not give a correct result for the splitting in the limiting case $Z_{1}=Z_{2}$. Thus, Power proposed to differentiate the half-sum of the $e Z_{1}$ - and $e Z_{2}$-energies with respect to the indices and take $T=T_{\mathrm{P}}$ (this result is presented in [1]). Finally, Ponomarev [10] noticed that the dependence of $\beta(E)$ on the energy should be considered when differentiating the $e Z_{1}$ - and $e Z_{2}$-energies leading to $T=T_{\mathrm{PKS}}$. The numerical values of preexponential factor, given by these formulae, differ from each other.

It is of interest to estimate the limits of applicability and practical accuracy of our asymptotic formula (12) for $\Delta E$ by its comparison with the results of numerical integration of a $Z_{1} e Z_{2}$ problem. In Table 1 we compare the values of $\Delta E$ in the $Z_{1} e Z_{2}$ system $\left(Z_{1}=1,4 \leqslant Z_{2} \leqslant 8\right)$ provided by our formula (12) with the analytical results $\Delta E_{\mathrm{PKS}}$ [1] and $\Delta E_{B}$ [11] as well as with the numerical calculations $\Delta E_{\text {num }}[12]$ ( Nlm are spherical quantum numbers in the limit of the united atom $(R=0)$ ). The comparison shows that the values of $\Delta E$ are quite close to the exact ones. The proximity of these results convincingly demonstrates the usefulness of the WKB method developed here.

Table 1. Adiabatic energy splittings $\Delta E$ at the quasicrossing points $R_{c}$ in the system $\left(p, e, Z_{2}\right)$

\begin{tabular}{ccccccc}
\hline$Z_{2}$ & $(N l m)-\left(N^{\prime} l^{\prime} m^{\prime}\right)$ & $R_{c}$ & $\Delta E(12)$ & $\Delta E_{\mathrm{PKS}}[1]$ & $\Delta E_{B}[11]$ & $\Delta E_{\mathrm{num}}[12]$ \\
\hline 4 & $(4,3,0)-(3,2,0)$ & 7.76 & $6.66 \times 10^{-2}$ & $6.56 \times 10^{-2}$ & - & $6.94 \times 10^{-2}$ \\
5 & $(5,4,0)-(4,3,0)$ & 12.9 & $4.07 \times 10^{-3}$ & $6.09 \times 10^{-3}$ & $4.16 \times 10^{-3}$ & $4.25 \times 10^{-3}$ \\
6 & $(6,5,0)-(5,4,0)$ & 21.4 & $2.40 \times 10^{-5}$ & $3.37 \times 10^{-5}$ & $2.41 \times 10^{-5}$ & - \\
7 & $(7,6,0)-(6,5,0)$ & 31.9 & $2.06 \times 10^{-8}$ & $2.44 \times 10^{-8}$ & $2.14 \times 10^{-8}$ & - \\
8 & $(8,7,0)-(7,6,0)$ & 44.3 & $3.04 \times 10^{-12}$ & $4.51 \times 10^{-12}$ & $2.88 \times 10^{-12}$ & - \\
\hline
\end{tabular}

Acknowledgements. This work is supported by the Ministry of Education and Science of the Russian Federation (Agreement No. 02.a03.21.0008) and the Ministry of Education, Science, Research, and Sport of the Slovak Republic (VEGA Grant No. 1/0345/17).

\section{References}

[1] I.V. Komarov, L.I. Ponomarev, and S.Yu. Slavyanov, Spheroidal and Coulomb Spheroidal Functions (Moscow: Nauka, 1976) [in Russian]

[2] I.V. Komarov, S.Yu. Slavyanov, J. Phys. B: At. Mol. Phys. 1, 1066 (1968)

[3] J.D. Power, Phil. Trans. Roy. Soc. London Ser. A 274, 663 (1973)

[4] T.M. Kereselidze, M.I. Chibisov, in Physics of Electronic and Atomic Collisions (LFTI, Leningrad, 1989) p. 160

[5] T.P. Grozdanov, R.K. Janev, V.Yu. Lazur, Phys. Rev. A 32, 3425 (1985)

[6] M. Hnatič, V.M. Khmara, V.Yu. Lazur, O.K. Reity, EPJ Web of Conf. 108, 02028 (2016)

[7] M. Hnatič, V.M. Khmara, V.Yu. Lazur, O.K. Reity, Theor. Math. Phys. 190, 345 (2017)

[8] O.B. Firsov, Zh. Eksp. Teor. Fiz. 21, 1001 (1951)

[9] P.J. Greenland, J. Phys. B: At. Mol. Phys. 11, 3563 (1978)

[10] L.I. Ponomarev, Sov. Phys. JETP 28, 971 (1969)

[11] A.A. Bogush and V.S. Otchik, J. Phys. A: Math. Gen. 30, 559 (1997)

[12] I.V. Komarov and N.F. Truskova, JINR Report No. P4-11445 (1978) 